\title{
Perspective
}

PERSPECTIVE Actualité en histoire de l'art

2| 2007

La Grande-Bretagne/Période moderne

\section{Stanisław Leszczyński, roi de Pologne et duc de Lorraine}

Bogna Kietlińska

\section{(2) OpenEdition}

1 Journals

Édition électronique

URL : http://journals.openedition.org/perspective/3847

DOI : 10.4000/perspective.3847

ISSN : 2269-7721

Éditeur

Institut national d'histoire de l'art

Édition imprimée

Date de publication : 30 juin 2007

Pagination : 392-395

ISSN : 1777-7852

Référence électronique

Bogna Kietlińska, «Stanisław Leszczyński, roi de Pologne et duc de Lorraine », Perspective [En ligne],

2 | 2007, mis en ligne le 31 mars 2018, consulté le 01 octobre 2020. URL : http://

journals.openedition.org/perspective/3847 ; DOI : https://doi.org/10.4000/perspective.3847

Ce document a été généré automatiquement le 1 octobre 2020. 


\title{
Stanisław Leszczyński, roi de Pologne et duc de Lorraine
}

\author{
Bogna Kietlińska
}

\section{RÉFÉRENCE}

Artur Badach éd., Stanislas, un roi de Pologne en Lorraine, (cat. expo., Nancy, Musée lorrain, 2004-2005), Versailles, ArtLys, 2005. 240 p., env. 100 fig. coul. ISBN-13 : 978-2854952339; $30 €$

Zamek Królewski éd., Stanisław Leszczyński. Król Polski ksiĘciem Lotaryngii [Stanisław Leszczyński. Roi de Pologne et duc de Lorraine], (cat. expo., Varsovie, Château royal de Varsovie, 2005), Varsovie, 2005. 280 p., 58 fig. n. et b., 187 fig. coul. ISBN :

83-7022-149-1; 55 zł.

Hanna Widacka, Z króla książż. Stanisław Leszczyński w grafice XVIII wieku ze zbiorów polskich i francuskich [Un roi devenu duc. Stanisław Leszczyński dans les gravures du XviII siècle issues des collections polonaises et françaises], Varsovie, 2006. 308 p. ; 130 fig. ISBN :

83-7009-477-5 ; 45 zł.

1 Stanisław Leszczyński (1677-1766) est un personnage singulier de l'histoire. Sa vie tumultueuse, riche en épisodes picaresques, lui a apporté une célébrité bien plus grande en France, en Suède et en Allemagne qu'en Pologne, où il a peu fait l'objet de recherches approfondies. Un des rares auteurs polonais qui s'est intéressé à lui est Edmund Cieślak, auteur de la seule biographie exhaustive sur le roi de Pologne ${ }^{1}$ jusqu'à récemment ainsi que d'autres travaux ${ }^{2}$. Dernièrement, on a pu observer un intérêt croissant des chercheurs pour ce personnage, dont témoigne le nombre croissant de publications en histoire et en histoire de l'art et d'expositions qui lui sont consacrées.

2 Stanisław Leszczyński a passé une partie de sa vie en Pologne et une autre en France, dans le duché de Lorraine et de Bar, les deux périodes étant séparées par une longue errance. En 1704, suite aux démarches de Charles XII et à une élection illégitime, il est couronné roi de Pologne mais ne le reste que très peu de temps car, après la défaite des 
forces suédoises contre la Russie, le trône revient à Auguste II de Saxe : Leszczyński est alors forcé à l'exil. La fortune lui sourit au moment du mariage de sa fille Marie avec le roi de France Louis XV. En 1733, soutenu cette fois-ci par les Français, Leszczyński tente à nouveau de monter sur le trône de Pologne. Toutefois, bien que sa deuxième élection se soit faite en toute légitimité, il doit se battre contre les Saxons qui ne veulent pas lui céder la couronne de Pologne. Quelques années plus tard, en 1737, il est nommé duc de Lorraine puis duc de Bar, deux provinces sur lesquelles pendant presque trente ans il régna en souverain éclairé, ce qui lui valut une grande estime et beaucoup de sympathie de la part de ses sujets. Leszczyński s'est principalement fait connaître comme un important mécène des arts et des sciences.

3 Le premier signe du renouveau de l'intérêt pour Stanisław Leszczyński en Pologne fut la grande exposition présentée au Château royal de Varsovie du 25 avril au 10 juillet 2005, en partenariat avec le Musée lorrain, le Musée des beaux-arts et la Bibliothèque municipale de Nancy dans le cadre de la Saison polonaise Nova Polska en France et de la célébration des deux cent cinquante ans de la Place Stanislas à Nancy. La version française de cette exposition, Stanislas, un roi de Pologne en Lorraine, s'est tenue à Nancy du 17 décembre 2004 au 21 mars 2005.

4 À l'occasion de ces deux manifestations culturelles, deux impressionnants catalogues ont été publiés ${ }^{3}$. Richement illustrés, ils contiennent, en plus des notices exhaustives sur chaque pièce exposée, des essais rédigés par des spécialistes français et polonais de la question, qui se penchent sur différents problèmes liés au roi Stanisław. La version française du catalogue contient 14 articles, parmi lesquels celui de Jerzy Gutkowski (absent de la version polonaise), "Devenir roi en Pologne», qui s'adresse plus particulièrement au lecteur français car il porte sur les élections royales en Pologne. Par ailleurs, la rédaction du catalogue français a fortement raccourci le texte de Józef Andrzej Gierowski, «Stanislas I Ir Leszczyński deux fois roi de Pologne ». Dans la version polonaise, l'article de Michelle Lespes, «Portraits de Stanislas ou pour une vision audelà du réel», a été remplacé par le texte de Hanna Widacka, consacré aux représentations de Stanisław Leszczyński dans les arts graphiques du XvIII ${ }^{e}$ siècle.

5 L'exposition française présentait 120 objets - dessins, tableaux, objets historiques et artisanat d'art. Il a été possible de voir entre autres de magnifiques portraits de Stanisław : Portrait de Stanislas, roi de Pologne et duc de Lorraine (après 1766), œuvre de Jean Girardet et l'extraordinaire tableau réalisé en 1727 par Jean-Baptiste Van Loo à l'occasion du mariage de Maria Leszczyńska avec Louis XV. Ils étaient accompagnés de ceux de Katarzyna Opalińska et de Maria Leszczyńska peints par Van Loo pour la même occasion. Outre cela, l'exposition présentait de très nombreux objets d'usage quotidien ayant appartenu au roi, plusieurs ouvrages de sa plume (entre autres Glos wolny wolność ubezpieczajĄcy ${ }^{4}$ ) et des lettres issues de sa correspondance privée.

6 Pour des raisons d'ordre logistique et administratif, certaines pièces issues des collections suédoises et françaises n'ont pas pu être montrées à Varsovie. L'exposition polonaise a donc été complétée par des portraits du roi provenant de différentes collections polonaises et par des gravures qui n'avaient pas été présentées en France. Toutes ces œuvres figurent dans la version polonaise du catalogue qui intègre également presque toutes les pièces non présentées à Varsovie. L'exposition de Varsovie réunissait 150 œuvres d'art. Les organisateurs polonais ont consacré beaucoup plus de place à la reine Maria Leszczyńska en lui réservant une salle à part. Ils ont également mis un peu plus l'accent sur la période polonaise de Stanisław et sur les 
relations entre la Pologne et la Lorraine. Parmi les œuvres majeures présentées uniquement à Varsovie, quelques toiles méritent de retenir plus spécialement l'attention :

- Le portrait de Stanisław Leszczyński peint d'après Gustaf Lundberg (?) provenant de la collection du Musée national de Varsovie, actuellement au Musée du Palais de Wilanów (ce tableau est une version réduite du portrait en pied de Leszczyński attribué à Gustaf Lundberg, issu de la collection du Musée du château de Versailles).

- Le tableau peint en 1733-1734 par Daniel Klein, issu de la collection du Château royal de Wawel. Il s'agit d'un portrait en buste de Stanisław Leszczyński, sans perruque, avec une grosse moustache. Le roi est vêtu d'une armure avec un manteau rouge doublé d'hermine posé sur les épaules. La représentation peut paraître assez étonnante, du fait de l'allure typiquement "polonaise » du monarque pour renouer avec ses anciennes effigies. Il s'agissait sans doute d'une démarche volontaire car, compte tenu de l'élection imminente de 1733, il cherchait à plaire à la noblesse polonaise.

- Un portrait d'un peintre de l'atelier d'Antoine Pesne, vers 1736. Le tableau fait partie de la collection du Musée Jerzy Dunin-Borkowski à Krośniewice. Le roi est présenté en buste, coiffé d'une longue perruque poudrée qui lui retombe sur la nuque et le dos où elle est nouée d'un ruban bleu. Il est vêtu d'un large manteau bleu foncé doublé d'hermine attaché par-devant au moyen d'une magnifique boucle, avec la plaque de l'Ordre du Saint-Esprit cousue sur l'épaule gauche. Le manteau est également orné des deux aigles polonais brodés de fils d'argent. La représentation est visiblement idéalisée et caractéristique des portraits de Pesne.

7 Si les deux expositions ont certainement mis en valeur l'homme politique qu'était Stanisław Leszczyński, elles ont également insisté sur l'intérêt pour l'art qu'il a cultivé durant toute sa vie et qui, à certaines époques, l'emportait même sur ses préoccupations politiques. En effet, le roi s'était engagé dans le domaine des arts non seulement à titre de mécène, mais aussi comme artiste, essentiellement à travers la peinture, et également comme conseiller lorsqu'il supervisait différentes réalisations architecturales et urbanistiques. Son intérêt pour l'architecture était sans doute le plus fort de tous ses intérêts artistiques, car il faisait partie des principales responsabilités du monarque idéal, titre auquel Leszczyński a toujours prétendu.

Les deux expositions ont bien montré la collaboration de Stanisław Leszczyński avec différents artistes. Fait caractéristique de sa démarche, il faisait appel non pas à des artistes parisiens ou versaillais mais à des artistes et artisans lorrains dont il devenait le mécène, contribuant ainsi à l'évolution de leur talent et de leur renommée. Parmi ceux qui doivent leur célébrité au roi Stanisław, on trouve Jean Lamour, auteur des magnifiques ouvrages en fer forgé qui ornent notamment la Place Royale de Nancy, et les sculpteurs Barthélemy Guibal et Paul-Louis Cyfflé. Au nombre des peintres les plus proches de Leszczyński, il faut compter Jean Girardet (1709-1778) que le roi fait nommer comme son peintre ordinaire par décret ducal du 10 mai 1758.

9 Si le roi trouve si facilement un langage commun avec les artistes, c'est parce que luimême peint et dessine en amateur. En plus des nombreux témoignages laissés par ses contemporains qui confirment la passion de Leszczyński pour la peinture, il existe plusieurs œuvres de sa main qui nous sont parvenues. Certaines ont été présentées dans le cadre des deux expositions. Le roi se consacre principalement à l'art du pastel et de la miniature, très en vogue à l'époque. Parmi les sujets récurrents on trouve des portraits qui constituent l'essentiel de son œuvre picturale, et des scènes religieuses. 
Un autre événement important a sans aucun doute été la parution du livre de Hanna Widacka $Z$ króla książż. Stanisław Leszczyński $w$ grafice XVIII wieku ze zbiorów polskich $i$ francuskich [Un roi devenu duc. Stanisław Leszczyński dans les gravures du XVIII siècle issues des collections polonaises et françaises] publié par la Biblioteka Narodowa (Varsovie, 2006). Une brève introduction précède un catalogue de 130 gravures représentant Leszczyński, dont près de la moitié provient de la Bibliothèque nationale de Pologne à Varsovie, les autres étant issues de différentes collections polonaises et de bibliothèques à Paris et à Nancy. La présentation des œuvres est organisée selon quelques grands groupes. L'étude s'ouvre par une série de 31 portraits de Stanisław en costume polonais, en buste ou en pied. Viennent ensuite 57 représentations du roi vêtu à la française, suivies des effigies de Leszczyński sur 19 médailles. Les deux dernières parties de l'ouvrage paraissent les plus intéressantes : la première présente 7 portraits de Stanisław dans des compositions historiques, allégoriques et symboliques liées, pour la plupart, à la première élection et au couronnement du roi, la deuxième réunissant 16 portraits dans des compositions historiques, allégoriques, symboliques et satiriques liées principalement à la deuxième élection et l'investiture du duché de Lorraine et de Bar. En plus de la présentation des gravures, le livre contient un important appareil scientifique, avec notamment le dictionnaire des artistes et des éditeurs de gravures.

11 Même si l'ouvrage de Hanna Widacka n'est pas une présentation exhaustive des œuvres graphiques consacrées à Leszczyński, il en révèle cependant l'impressionnante diversité. Une autre grande qualité de ce livre est sans doute la présence de représentations historiques, allégoriques et surtout, satiriques qui permettent de mieux appréhender non seulement la personnalité du roi mais aussi les opinions et l'atmosphère de l'époque.

\section{NOTES}

1. Edmund Cieślak, Stanisław Leszczyńskim, Wrocław, 1994.

2. Edmund Cieślak, $W$ obronie tronu króla Stanisława Leszczyńskiego [La défense du trône du roi Stanisław Leszczyński], Gdańsk, 1986. Signalons, parmi les autres travaux du même auteur, l'édition d'un texte de Leszczyński particulièrement intéressant racontant sa propre fuite de Gdańsk à Kwidzyń, et accompagné d'un commentaire d'E. Cieslak relatif à la fuite du roi : Stanisław Leszczyński, Opis ucieczki z Gdańska do Kwidzynia, Olsztyn, 1988.

3. Artur Badach éd., Stanislas, un roi de Pologne en Lorraine, (cat. expo., Nancy, Musée lorrain, 2004-2005), Versailles, 2004; Zamek Królewski éd., Stanisław Leszczyński. Król Polski księciem Lotaryngii [Stanisław Leszczyński. Roi de Pologne et duc de Lorraine], (cat. expo., Varsovie, Château royal de Varsovie, 2005), Varsovie, 2005.

4. Stanisław Leszczyński, La voix libre du citoyen, ou observations sur le gouvernement de Pologne, Nancy, 1733 (Paris, 1749), 2 vol. 
INDEX

Mots-clés : mécène, portrait, représentation, expositions, peintre amateur

Index géographique : France, Lorraine, Pologne, Europe

Keywords : patron, portrait, representation, exhibitions, painter

Index chronologique : 1700 